\title{
DETERMINATION OF SERUM VISININ LIKE PROTEIN-1 AND ITS POTENTIAL FOR THE DIAGNOSIS OF BRAIN INJURY DUE TO THE STROKE - A PILOT STUDY
}

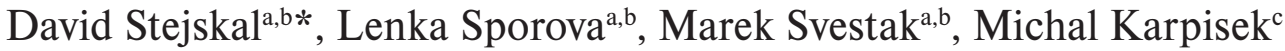

\author{
${ }^{a}$ Department of Laboratory Medicine and Department of Medicine, Prostejov Hospital, Central Moravian Hospital County \\ Inc., Czech Republic \\ ${ }^{b}$ Department of Medical Chemistry and Biochemistry, Faculty of Medicine and Dentistry, Palacky University Olomouc \\ c Department of Human Pharmacology and Toxicology, Faculty of Pharmacy, University of Veterinary and Pharmaceutical \\ Sciences Brno \\ E-mail: david.stejskal@nemsne.cz
}

Received: February 15, 2011; Accepted with revision: July 11, 2011

Key words: VILIP-1/Visinin like protein 1/Stroke/ELISA

Background. The current diagnosis of stroke relies on clinical examination by a physician supplemented by various neuroimaging techniques. A single set or multiple sets of blood biomarkers that could be used in acute settings to diagnosis stroke, differentiate between stroke types, and ideally predict an initial/recurring stroke would be extremely valuable. The diagnosis of stroke is currently hampered by delay due to lack of a suitable tool for rapid, accurate and analytically sensitive biomarker - based testing. There is a clear need for further assay development and clinical validation in this area (acute stroke setting) in order to improve patient outcomes and quality of life. Visinin like protein 1 (VILIP-1) is a newly discovered CNS-abundant protein which has shown promise in experimental studies, for early stroke diagnosis. However, to date there is no clinical study that has measured VILIP-1 in sera as a marker of stroke.

Aim. To develop an assay for the determination of VILIP-1 in human serum, and to investigate its clinical relevance as a marker of ischemic stroke.

Design and methods. A new sandwich ELISA was developed, introduced and clinically tested. Mean spiking recovery was $98 \%$. The mean recovery for dilution linearity was $93 \%$. The limit of detection of the assay was $0.01 \mathrm{mcg} / \mathrm{l}$; the intraassay and interassay coefficient of variation (CV) were always less than $10 \%$. The study was approved by the Ethics Commission of the Hospital Šternberk, Czech Republic. A total of 17 healthy individuals ( 9 men and 8 women, age $64.0 \pm 13.0$ ) and 16 individuals with ischemic stroke ( 10 men and 6 women, age $63.0 \pm 11.5$ ) were recruited for our study. The criteria of stroke were proposed by the National Czech Standard. All individuals had blood samples drawn, and VILIP-1 analysis and CT and/or MRI were performed.

Results. VILIP-1 serum level significantly differentiated healthy subjects from patients with stroke $(\mathrm{P}<0.01)$. All individuals with stroke had VILIP-1 serum values higher than $>0.05 \mathrm{mcg} / 1$, healthy had values below this value. The diagnostic efficacy of serum VILIP-1 was very significant (sensitivity $100 \%$, specificity $100 \%$ at $0.093 \mathrm{mcg} / 1 \mathrm{VILIP}-1$ serum values, AUC 1.0 (CI 0.93-1.0, $\mathrm{P}<0.01$ ), Chi-squared in the frequency table was 33 ( $\mathrm{P}<0.01)$.

Conclusion. We have introduced a new analytical tool for the study of VILIP-1. Our results support the hypothesis that serum VILIP-1 may be associated with ischemic stroke. The ELISA VILIP-1 assay offers a new research tool for the diagnosis and pathophysiology of stroke and other CNS diseases.

\section{INTRODUCTION}

Stroke is the third leading cause of morbidity and mortality in the Western world, following cardiovascular diseases and cancer ${ }^{1}$. Globally there are more than 50 million stroke and transient ischemic attack (TIA) survivors. More than 1 in 5 survivors will suffer a subsequent stroke in the following 5 years ${ }^{1}$. Blacks have twice the risk of stroke compared with whites, and women are at a greater risk for stroke than men ${ }^{1}$. Recent estimates suggest the worldwide economic cost of stroke to be approximately $\$ 68.9$ billion, including direct and indirect cost $^{2}$.

Broadly, stroke is classified as ischemic or hemorrhagic, with ischemic stroke accounting for approximately $85 \%$ of the total number ${ }^{2,3}$. Ischemic stroke is primarily caused by either intracranial thrombosis or extracranial embolism $^{3}$. Clinical diagnosis and management of stroke are predicated on obtaining an accurate medical history and a thorough physical assessment of the patient. The diagnostic accuracy of stroke has been shown to have a sensitivity of $92 \%$ by primary care physicians routinely exposed to suspected stroke victims ${ }^{4}$, but is less reliable in physicians with less experience or confidence ${ }^{5}$. Rapid assessment of acute stroke victims is critical for determining eligibility for thrombolytic therapy. To assist with diagnostic consistency among physicians, the 42-point National Institutes of Health Stroke Scale (NIHSS) scale was designed to be completed within 5 to $8 \mathrm{~min}^{6,7}$. Neuroimaging remains the only tool available for differentiating between ischemic stroke and intracerebral hemorrhage as the symptoms 
Table 1. Proteins resulting from expression of genes in CNS.

\begin{tabular}{|l|l|}
\hline VSNL1 & Visinin-like 1 \\
\hline SNAP25 & Synaptosomal-associated protein \\
\hline GAD1 & Glutamate decarboxylase 1 (brain, Mr 67 000) \\
\hline MOBP & Myelin-associated oligodendrocyte basic protein \\
\hline SYT1 & Synaptotagmin I 6857 \\
\hline TUBB4 & Tubulin_4 10382 \\
\hline FEZ1 & Fasciculation and elongation protein_1 (zygin I) \\
\hline GLRB & Glycine receptor__2743 Hs0.32973 14 726.1 \\
\hline VMP & Vesicular membrane protein p24 \\
\hline OLFM1 & Olfactomedin 1 \\
\hline ZIC1 & Zic family member 1 (odd-paired homolog, Drosophila) \\
\hline PACSIN1 & Protein kinase C and casein kinase substrate in neurons 1 \\
\hline PLP1 & Proteolipid protein 1 (Pelizaeus-Merzbacher disease,spastic paraplegia \\
\hline INA & Internexin neuronal intermediate filament protein \\
\hline SLC32A1 & Solute carrier family 32 (GABAb vesicular transporter), member 1 \\
\hline SERPINI1 & Serine (or cysteine) proteinase inhibitor, clade I (neuroserpin) \\
\hline NNAT & Neuronatin 4826 \\
\hline GABRG2 & GABA A receptor_2 \\
\hline MP2 & Vesicle-associated membrane protein 2 (synaptobrevin 2) \\
\hline NRGN & Neurogranin (protein kinase C substrate, RC3) \\
\hline
\end{tabular}

of the 2 conditions show substantial overlap. Diagnostic criteria for stroke do not use specific blood biomarkers, instead relying solely on clinical assessment and interpretation of radiological findings. The primary goal for the initial neurological imaging studies is to differentiate between hemorrhagic and ischemic stroke or exclude stroke mimics in a timely manner. Many important features gained from brain imaging ultimately help guide treatment decisions and options, which include detecting early infarction and determining the location and degree of infarct and vascular distribution of the lesions responsible for the stroke. Computed tomography (CT) and magnetic resonance imaging (MRI) are routinely used in the initial acute assessment of stroke victims ${ }^{8-10}$.

Current diagnosis of stroke relies on physician clinical examination and is further supplemented with various neuroimaging techniques. Unfortunately, there are no known individual blood biomarkers that can be targeted for routine use in the setting of acute stroke diagnosis, differentiation, or risk prediction, even if multimarker panels have been developed and investigated in an attempt to improve the diagnostic sensitivity and specificity of individual biomarkers. There are several promising specific markers in blood suitable for diagnostic of CNS disease (S-100B, NSE, GFAP, secretagogin, N-methyl-D-aspartic acid (NMDA) receptor antibodies, PARK 7, nucleoside diphosphate kinase A) or estimating predisposition for CNS disease (lipoprotein-associated phospholipase A2, asymmetric dimethylarginine, matrix metalloproteinase 9, C-reactive protein, VCAM-1, A-FABP, BNP).

It is hypothesized that other cytoplasmic proteins of neural cells, which predominantly occur in cells and are released into circulation as a consequence of its destruction, might be a target for further research. Currently this list includes 20 proteins, which are found in the brain (Table 1), including VILIP-1 (ref. ${ }^{1}$ ).
The visinin-like protein (VSNL) subfamily, including VILIP-1, VILIP-2, VILIP-3, hippocalcin, and neurocalcin delta, constitutes a highly homologous subfamily of neuronal calcium sensor proteins. Comparative studies have shown that VSNLs are expressed predominantly in the brain with restricted expression patterns in various subsets of neurons ${ }^{11}$.

VILIP-1 is found in all CNS locations and penetrates into CSF after destruction of brain cells ${ }^{1}$. Concentration of VILIP-1 protein has not yet been determined in blood or CSF. Only a few papers have described the determination of gene expression (mRNA), but these did not concern neurological diagnoses.

The aim of our work was to find a relationship between the concentration of serum VILIP 1 and other markers of damage to brain cells in individuals with ischemic stroke.

\section{MATERIALS AND METHODS}

\section{VILIP-1 immunoassay development}

We established and evaluated a sandwich ELISA for the quantitative determination of VILIP-1 in human serum and CSF. The assay employs specific rabbit polyclonal anti-human VILIP-1 antibody (Biovendor, Brno, Czech Republic), which was raised against recombinant human VILIP-1 (Biovendor) as an antigen. Antibody was purified on a column with immobilized recombinant human VILIP-1 and was used as the capture and detection antibody.

The sandwich ELISA employs specific rabbit polyclonal anti-human VILIP-1 antibody provided by Biovendor, and coated on microtiter wells (NUNC, Maxisorp): $100 \mu \mathrm{l} / \mathrm{well}, 1 \mu \mathrm{g} / \mathrm{ml}$ in $0.1 \mathrm{M}$ carbonate buffer overnight at $4{ }^{\circ} \mathrm{C}$. The plate was washed once with TBS-Tw $(0.05 \mathrm{M}$ Tris-HCl; $0.15 \mathrm{M} \mathrm{NaCl}$; pH 7.2; 0.05\%) (w/v) Tween 20 on 
Table 2. Laboratory basic results of healthy control group (0) vs. patiens with stroke (1).

\begin{tabular}{|c|c|c|c|c|c|c|c|}
\hline & \multicolumn{3}{|c|}{0} & \multicolumn{4}{|c|}{1} \\
\hline & Mean & SD & Median & Differences & Mean & SD & Median \\
\hline CSF_ALB & 446.4 & 368.6 & 355.9 & $\mathrm{P}=0.08, \mathrm{NS}$ & 286.8 & 150.1 & 232.6 \\
\hline CSF_APOA & 5.9 & 3.0 & 5.1 & $\mathrm{P}=0.20, \mathrm{NS}$ & 5.2 & 3.7 & 4.0 \\
\hline CSF_ApoB & 1.6 & 0.4 & 1.5 & $\mathrm{P}=0.23, \mathrm{NS}$ & 1.8 & 1.6 & 1.5 \\
\hline CSF_BAM & 795.6 & 225.4 & 799.7 & $\mathrm{P}=0.18, \mathrm{NS}$ & 876.7 & 277.7 & 893.5 \\
\hline CSF_CLUS & 7008.4 & 3921.6 & 6151.0 & $\mathrm{P}=0.71, \mathrm{NS}$ & 6430.5 & 2238.1 & 7297.5 \\
\hline CSF_CRP & 129.8 & 309.6 & 14.7 & $\mathrm{P}=0.95, \mathrm{NS}$ & 88.4 & 222.9 & 18.3 \\
\hline CSF_CysC & 5.2 & 0.8 & 5.1 & $\mathrm{P}=0.71, \mathrm{NS}$ & 5.5 & 2.1 & 5.7 \\
\hline CSF_FIBR & 4.4 & 0.9 & 4.1 & $\mathrm{P}=0.93, \mathrm{NS}$ & 4.3 & 0.5 & 4.4 \\
\hline CSF_Hap & 4.5 & 8.3 & 2.4 & $\mathrm{P}=0.06, \mathrm{NS}$ & 2.0 & 2.4 & 0.9 \\
\hline CSF_IGA & 5.4 & 3.5 & 4.7 & $\mathrm{P}=0.51, \mathrm{NS}$ & 6.1 & 3.3 & 6.4 \\
\hline CSF_IGG & 46.4 & 24.7 & 39.5 & $\mathrm{P}=0.39, \mathrm{NS}$ & 53.6 & 23.2 & 63.8 \\
\hline CSF_IGM & 2.1 & 5. & 0.6 & $\mathrm{P}=0.95, \mathrm{NS}$ & 0.8 & 0.6 & 0.6 \\
\hline CSF_ORM & 10.5 & 7.0 & 8.7 & $\mathrm{P}=0.24, \mathrm{NS}$ & 8.5 & 6.2 & 6.9 \\
\hline CSF_PREA & 18.6 & 2.0 & 18.1 & $\mathrm{P}=0.58, \mathrm{NS}$ & 18.9 & 2.1 & 18.5 \\
\hline CSF_TAU & 240.3 & 85.5 & 231.0 & $\mathrm{P}=0.15, \mathrm{NS}$ & 399.3 & 382.4 & 282.0 \\
\hline S_ALB & 42.7 & 4.9 & 43.0 & $\mathrm{P}=0.09, \mathrm{NS}$ & 39.9 & 5.6 & 40.0 \\
\hline S_APOA & 1.2 & 0.2 & 1.2 & $\mathrm{P}=0.30, \mathrm{NS}$ & 1.1 & 0.3 & 1.1 \\
\hline S_APOB & 1.0 & 0.3 & 1.0 & $\mathrm{P}=0.89, \mathrm{NS}$ & 1.0 & 0.3 & 1.0 \\
\hline S_CLUS & 6954.5 & 2148.2 & 6897.4 & $\mathrm{P}=0.49, \mathrm{NS}$ & 7123.5 & 2432.0 & 7011.9 \\
\hline S_CRP & 34.7 & 73.9 & 4.3 & $\mathrm{P}=0.29, \mathrm{NS}$ & 12.5 & 19.1 & 5.4 \\
\hline S_CYST & 0.9 & 0.2 & 0.9 & $\mathrm{P}=0.15, \mathrm{NS}$ & 1.1 & 0.2 & 1.0 \\
\hline S_HP.. & 1.8 & 1.2 & 1.6 & $\mathrm{P}=0.15, \mathrm{NS}$ & 1.3 & 0.5 & 1.3 \\
\hline S_IGA & 2.1 & 0.7 & 2.0 & $\mathrm{P}=0.10, \mathrm{NS}$ & 3.0 & 1.2 & 2.8 \\
\hline S_IGG & 8.9 & 2.8 & 8.2 & $\mathrm{P}=0.30, \mathrm{NS}$ & 11.4 & 4.7 & 11.8 \\
\hline S_IGM & 1.1 & 0.5 & 1.0 & $\mathrm{P}=0.61, \mathrm{NS}$ & 1.3 & 1.0 & 0.9 \\
\hline S_PREA & 0.3 & 0.1 & 0.3 & $\mathrm{P}=0.78, \mathrm{NS}$ & 0.3 & 0.1 & 0.3 \\
\hline S_ORM & 1.1 & 0.4 & 1.0 & $\mathrm{P}=0.18, \mathrm{NS}$ & 0.9 & 0.3 & 0.9 \\
\hline S_VILIP-1 & 0.03 & 0.03 & 0.02 & $P<0.01, V^{*}$ & 1.8 & 3.6 & 0.8 \\
\hline Ind_Alb & 10.6 & 8.6 & 7.8 & $\mathrm{P}=0.13, \mathrm{NS}$ & 7.5 & 5.0 & 6.0 \\
\hline Ind_ApoA & 5.1 & 2.6 & 4.6 & $\mathrm{P}=0.24, \mathrm{NS}$ & 5.0 & 4.6 & 3.6 \\
\hline Ind_ApoB & 1.7 & 0.6 & 1.5 & $\mathrm{P}=0.91, \mathrm{NS}$ & 2.2 & 2.6 & 1.5 \\
\hline Ind_CLUS & 0.9 & 0.2 & 0.9 & $\mathrm{P}=0.84, \mathrm{NS}$ & 0.9 & 0.6 & 0.8 \\
\hline Ind_CRP & 6.9 & 6.4 & 4.7 & $\mathrm{P}=0.73, \mathrm{NS}$ & 9.1 & 8.9 & 4.0 \\
\hline Ind_CYST & 5.7 & 1.7 & 5.8 & $\mathrm{P}=0.57, \mathrm{NS}$ & 5.1 & 2.0 & 5.3 \\
\hline Ind_HP & 1.9 & 1.8 & 1.3 & $\mathrm{P}=0.90, \mathrm{NS}$ & 2.0 & 3.7 & 0.9 \\
\hline Ind_IGA & 2.5 & 1.5 & 2.1 & $\mathrm{P}=0.81, \mathrm{NS}$ & 2.1 & 1.1 & 2.0 \\
\hline Ind_IGG & 5.7 & 3.6 & 4.8 & $\mathrm{P}=0.76, \mathrm{NS}$ & 4.9 & 2.6 & 4.8 \\
\hline Ind_IGM & 1.4 & 2.4 & 0.7 & $\mathrm{P}=0.86, \mathrm{NS}$ & 0.7 & 0.5 & 0.5 \\
\hline Ind_PREA & 81.6 & 40.1 & 67.6 & $\mathrm{P}=0.69, \mathrm{NS}$ & 82.7 & 38.2 & 68.5 \\
\hline Ind_ORM & 9.5 & 4.4 & 8.0 & $\mathrm{P}=0.72, \mathrm{NS}$ & 9.1 & 5.2 & 8.2 \\
\hline Ind_VILIP-1 & 4.73 & 6.50 & 2.60 & $\mathrm{P}<0.01, \mathrm{VS}^{*}$ & 0.20 & 0.27 & 0.07 \\
\hline Csf_VILIP-1 & 0.07 & 0.05 & 0.07 & $\mathrm{P}<0.01, \mathrm{VS}^{*}$ & 0.16 & 0.15 & 0.10 \\
\hline
\end{tabular}

CSF_x = analysed in CSF; $\quad$ S_x = analysed in sera; $\mathrm{SD}=$ standard deviation; $\mathrm{ALB}=$ albumin; $\mathrm{APOA}=\mathrm{ApoAI} ; \mathrm{ApoB}=\mathrm{ApoB} ; \mathrm{BAM}=\mathrm{beta}$ a,mylpoid; tau = phosphorylated tau protein; clus = clusterin; $\mathrm{CRP}=\mathrm{C}$-reactive protein; $\mathrm{CysC} / \mathrm{CYST}=$ cystatin $\mathrm{C}$; Fibr $=$ fibrinogen; Hap = haptoglobin; IGA = immunoglobulin A; IGG = immunoglobulin G; IGM = immunoglobulin M; ORO = orosomucoid; PREA = prealbumin; VILIP-1 $=$ Visinin like protein $\mathrm{I}, \mathrm{P}=$ probability index, $\mathrm{NS}=$ non signifiant, VS $*$ very significant, Ind $=\mathrm{CSF} / \mathrm{S}$ ratio

the Columbus washer (Tecan). Non-specific binding sites were blocked with $200 \mu \mathrm{l} /$ well $4 \%$ saccharose, $0.5 \%$ bovine serum albumin in TBS for $30 \mathrm{~min}$ at $25^{\circ} \mathrm{C}$. After aspiration, diluted samples (serum or plasma samples diluted 3-fold with $1.4 \%$ casein buffer in a $0.15 \mathrm{M}$ PBS (0.12 M
$\mathrm{NaCl}, 0.03 \mathrm{M}$ sodium phosphate, buffer $\mathrm{pH}$ 7.3) or standards were pipetted in duplicates $100 \mu \mathrm{l} /$ well. The plate was incubated for $1 \mathrm{~h}$ at $25^{\circ} \mathrm{C} / \mathrm{RT}$. After five washes with TBS-Tw, $100 \mu \mathrm{l} /$ well of biotin-labelled rabbit polyclonal anti-human VILIP-1 antibody (labelling with the kit from 
Table 3. Freqency chart - VILIP-1 values divided by quartiles and diagnosis of stroke (Chi square $33, \mathrm{P}<0.01$ ).

\begin{tabular}{|c|c|c|c|c|c|}
\hline \multirow[b]{2}{*}{ Diagnosis } & \multicolumn{4}{|c|}{ Quartiles of S_VILIP-1 } & \multirow[t]{2}{*}{ SUM } \\
\hline & 1 & 2 & 3 & 4 & \\
\hline 0 & 17 & 0 & 0 & 0 & $17(51.5 \%)$ \\
\hline 1 & 0 & 3 & 6 & 7 & $16(48.5 \%)$ \\
\hline SUM & $\begin{array}{c}17 \\
(51.5 \%)\end{array}$ & $\begin{array}{c}3 \\
(9.1 \%)\end{array}$ & $\begin{array}{c}6 \\
(18.2 \%)\end{array}$ & $\begin{array}{c}7 \\
(21.2 \%)\end{array}$ & 33 \\
\hline
\end{tabular}

Diagnosis $0=$ healthy, Diagnosis $1=$ stroke

Pierce) was added and the plate was incubated for $1 \mathrm{~h}$ at $25^{\circ} \mathrm{C}$. Following five washes, $100 \mu \mathrm{l} /$ well of streptavidinHRP conjugate (Amdex) was added and the plate was incubated for $1 \mathrm{~h}$ at $25^{\circ} \mathrm{C}$. After washing, $100 \mu \mathrm{l} /$ well of TMB substrate (KPL) was then added and the plate was incubated for another $10 \mathrm{~min}$ at $25^{\circ} \mathrm{C}$. The reaction was stopped with $100 \mu \mathrm{l} /$ well of sulfuric acid $(0.2 \mathrm{M})$. The developed colour was determined by reading the plate on the microplate reader Biotek EL808 at a wavelength of $450 \mathrm{~nm}$.

Recombinant VILIP-1 provided by Biovendor was used as the standard for the assay. The protein content of recombinant VILIP-1 was determined by the BCA method (Sigma-Aldrich) and its purity confirmed by SDS PAGE (data not shown). Standards were prepared at concentrations of $6,3,1.5,0.6,0.15,0.06$ in casein buffer and $100 \mu 1$ was directly pipetted into the wells.

To validate the reliability of the assay, we tested the precision and the accuracy of the assay. To analyze the spiking recovery, human serum samples from two subjects with baseline VILIP-1 levels of 0.8 and $0.3 \mu \mathrm{g} / 1$ were spiked with increasing amounts of recombinant protein $(+0.5,+1.0$ and $+2.0 \mu \mathrm{g} / 1)$ and assayed. The mean recovery was $98 \%$. Moreover, we tested human serum samples from another two subjects with baseline VILIP-1 levels of 2.1 and $1.8 \mu \mathrm{g} / 1$ for dilution linearity. The mean recovery was $93 \%$. The limit of detection of the assay was $0.01 \mu \mathrm{g} / 1$; the intraassay and interassay coefficient of variation $(\mathrm{CV})$ were always less than $10 \%$.

\section{Study subjects}

The study was approved by the Ethics Commission of ŠternberkHospital, Czech Republic. A total of 17 healthy individuals ( 9 men and 6 women, age 64.0 113.0 ) and 16 individuals with stroke ( 10 men and 6 women, age $63.0 \pm 11.5$ ) were recruited for our study.

The criteria of stroke were proposed by a 42-point National Institutes of Health Stroke Scale (NIHSS) scale. Additionally, serum and CSF analysis, and examination by CT and/or MRI was carried out in all individuals.

\section{Sampling and data measurement}

Clinical measurement (systolic and diastolic pressures, body mass indexes, patient 's history) and laboratory fasting analyses were performed. Blood and CSF samples were drawn under aseptic precautions from vena cubiti or spinal canal; sera after a five-minute rest in the half-sitting

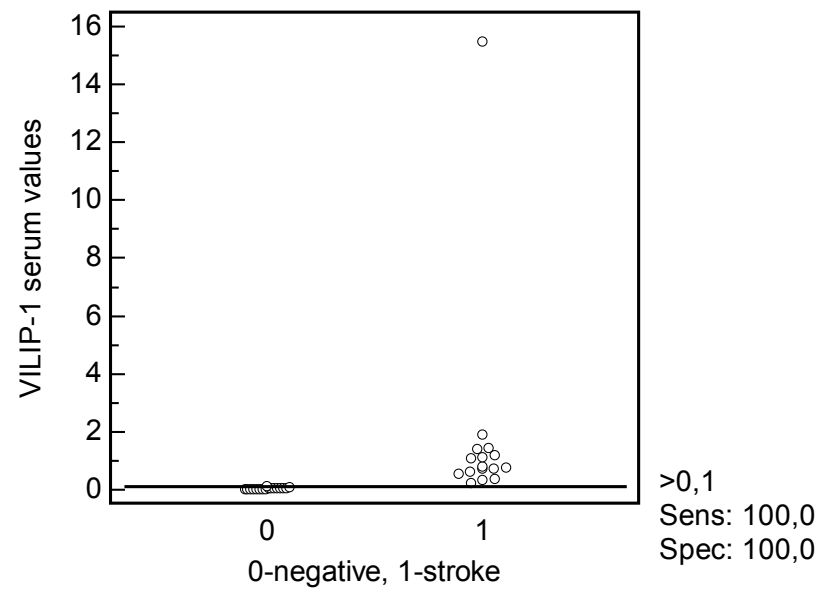

Fig. 1. ROC Dot chart, Serum VILIP-1 and individuals with stroke $(\mathrm{Dg}=0)$ vs. Healthy $(\mathrm{Dg}=1)$.

position, CSF after a five-minute rest in the prone lying position. Biological material was drawn within 3 hours following the first symptoms of CNS disease. Serum and CSF samples were separated in a cooled centrifuge at $3000 \mathrm{~g}$ for $20 \mathrm{~min}$ and immediately analyzed for glucose, natrium, potassium, chloride, total protein, cystatin $\mathrm{C}$, albumin, Immunoglobulin $\mathrm{G}$, Immunoglobulin $\mathrm{A}$, Immunoglobulin M, Apolipoprotein A, Apolipoprotein B, C-reactive protein (all Siemens, Advia 1650 and BN II). VILIP-1 serum and CSF level were determined by the assay presented above and clusterin serum and CSF level were determined by a commercially available ELISA kit (Biovendor, biorobot Dynex, 4DSX) in serum samples stored at $-80^{\circ} \mathrm{C}$. CSF/serum indexes and intrathecal synthesis of immunoglobulines were calculated according to the previously published formula ${ }^{12}$.

\section{Statistical analysis}

The data were processed by means of the software Medcalc (Medcalc, Mariakerke, Belgium). The value $\mathrm{p}<0.05$ was considered as statistically significant. Because of anomalous data distribution of evaluated parameters, Spearman's correlation coefficients were used to establish the association between VILIP-1 levels and the other parameters. Comparison of VILIP-1 serum values between subjects with and without stroke was performed by Mann-Whitney test according to the data distribution. All data are presented as medians and/or means \pm standard deviation. 


\section{RESULTS}

\section{Performance characteristics of human VILIP-1 ELISA}

The limit of detection of the assay was $0.01 \mu \mathrm{g} / 1$ and the limit of quantification was $0.02 \mu \mathrm{g} / \mathrm{l}$. The intra-assay coefficient of variation $(\mathrm{CV})$ was determined by replicate analysis $(\mathrm{n}=8)$ of four serum (CSF) samples at concentration $0.2(0.1), 0.8(1.3)$ and $1.7(1.5) \mu \mathrm{g} / 1$. The intraassay $\mathrm{CV}$ ranged from 3.2 to $7.1 \%$. The inter-assay $\mathrm{CV}$ was determined by duplicate measurement of serum (CSF) samples at baseline concentration $0.1(0.2), 0.5(0.6)$, $0.9(1.0)$, and $1.9(1.6) \mu \mathrm{g} / \mathrm{l}$ in separate assays $(\mathrm{n}=8)$. The inter-assay CV range was $5.8-9.9 \%$. To analyze the spiking recovery, human serum (CSF) samples from two subjects with baseline VILIP-1 levels of $0.3(0.4)$ and 0.8 (1.0) $\mu \mathrm{g} / 1$ were spiked with increasing amounts of recombinant protein $(+0.5,+1.0$ and $+2.0 \mu \mathrm{g} / \mathrm{l})$ and assayed. The mean recovery was $98 \%$ (99\%). Dilution linearity was determined in serum (CSF) samples from another two subjects with baseline VILIP-1 levels of 1.7 and $2.1 \mu \mathrm{g} / 1$, samples were serially diluted in assay dilution buffer. The mean recovery was $93 \%(95 \%)$.

\section{Clinical Data Analysis}

The study analyzed 33 subjects, of whom 17 were in good health while 16 probands suffered from stroke (average NIH stroke scale system 15.3; CT or/and MRI scan was typical from ischemia). We found no significant difference in VILIP-1 concentration between men and women in either serum or CSF in the group of healthy subjects (serum VILIP-1 in men and women: 0.03 vs. $0.02 \mu \mathrm{g} / 1, \mathrm{P}=0.8$; CSF VILIP-1 in men and women: 0.08 vs. $0.06 \mu \mathrm{g} / 1, \mathrm{P}=0.55)$ nor in the group of patients with ischemic stroke (serum VILIP-1 in men and women: 0.64 vs. $2.5 \mu \mathrm{g} / 1, \mathrm{P}=0.35$; CSF VILIP-1 in men and women: 0.23 vs. $0.12 \mu \mathrm{g} / 1, \mathrm{P}=0.07)$. However, we observed a significant difference between healthy subjects and probands with ischemic stroke (serum VILIP-1 in healthy subjects and subjects with stroke: 0.03 vs. $1.78 \mu \mathrm{g} / 1, \mathrm{P}<0.01$; CSF VILIP-1 in healthy subjects and subjects with ischemic stroke: 0.07 vs. $0.16 \mu \mathrm{g} / 1, \mathrm{P}<0.01$; (Table 2, Fig. 1). Clinical sensitivity and specificity of serum VILIP-1 for stroke diagnosis were $100 \%$.

We also prepared a frequency table of VILIP-1 (VILIP-1 divided by quartiles and diagnosis of ischemic stroke). Differences were significant (Chi square 33, $\mathrm{P}<0.01$ ) (Table 3).

Serum VILIP-1 level correlated with CSF ApoAI $(\mathrm{r}=-23 . \mathrm{P}=0.02)$, index CSF/S ApoAI $(\mathrm{r}=-0.23, \mathrm{P}=0.04)$ and CSF VILIP-1 $(\mathrm{r}=0.4, \mathrm{P}<0,01)$.

\section{DISCUSSION}

Current diagnosis of stroke relies on clinical examination by a physician, supplemented with various neuroimaging techniques. A single set or multiple sets of blood biomarkers that could be used in an acute setting to diagnosis stroke, differentiate between stroke types, and ideally predict an initial/recurring stroke would be extremely valuable.

In recently published reviews, 21 studies that evaluated 58 single biomarkers and 7 panels of several biomarkers were examined ${ }^{13,14}$. High diagnostic efficacy was demonstrated for the majority of the biomarkers when used alone; however, there were major limitations to the study designs and reporting that prevent recommendation of a specific biomarker for clinical use. Common study flaws noted in the reviews included small sample sizes, poor choice of reference standard, poor selection of control population, unclear diagnostic cutoff points, and overall lack of analytical characterization and clinical validation of the proposed biomarkers ${ }^{15}$.

VILIP-1 belongs to the family of NCS proteins involved in many physiological processes, such as pain modulation, neuropathological, myocardial processes and carcinogenesis ${ }^{16,17}$. At a molecular level, these proteins play a role in controlling neuronal function. Outside the brain, VILIP-1 occurs in the pancreas where it acts as a repressor gene for insulin, thereby increasing insulin secretion after administration of carbohydrate. For tumors, VILIP-1 acts as a tumor suppressor gene inhibiting cell proliferation, adhesion and invasiveness. Its expression is reduced in certain types of cancer. VILIP-1 expression has also been described in the heart, and it regulates the Natriuretic receptor B (reduces effect of braun natriuretic peptide) (ref. ${ }^{16}$ ). Its overexpression leads to enhanced aChR-dependent inhibitory postsynaptic current generation by interneurons ${ }^{17}$.

We developed, evaluated and validated (CE mark certification in progress) a new and simple ELISA sandwich method for determination of VILIP-1. It should be noted that individuals with stroke disease have increased levels of VILIP-1 in both serum and CSF. Moreover, we confirmed for the first time that VILIP-1 protein may be an independent marker of cerebral injury.

This assumption, however, should be verified in larger studies in individuals with various CNS diseases using certified standard diagnostic kits.

The major limitation of this study was the selection of control group. Since healthy subjects were selected, the comparison with stroke patients did not allow us to estimate the accuracy (sensitivity and specificity) for stroke as opposed to other CNS conditions. It is possible that Visinin like protein-1 may be increased in other CNS diseases which would not allow its use as a marker for differentiating stroke, neurodegeneration, neurodestruction, etc.

The authors are aware that the number of patients in this study is small. However, there are practical difficulties in obtaining patients with suspected ischemic stroke who undergo lumbar puncture. However, the main objective was to investigate the pathophysiological relationships between VILIP-1 and the other parameters and, second to determine links between brain damage and this marker. In effect this was only a pilot study to investigate fundamental relationships that have not yet been published. Whether VILIP-1 is an independent and specific indicator 
of CNS damage or an independent indicator of ischemic stroke cannot be answered by the present study due to small sample size. For many years, also discussed has been the timing of sampling. This study established that the sampling has to be done within $3 \mathrm{~h}$ of onset of symptoms. Overall, our results show that VILIP-1 is a promising indicator as a new marker of CNS damage.

In conclusion, this study introduces a new analytical tool for the study of VILIP-1. Our results support the hypothesis that serum VILIP-1 may be associated with brain disease. Future studies evaluating novel stroke biomarkers should answer questions that address their unique clinical contribution in the diagnosis, management, and risk prediction of brain disease.

The authors declare no conflicts of interests.

\section{ACKNOWLEDGEMENT}

Supported by the grant No. MSM 6198959216.

\section{REFERENCES}

1. Saenger AK, Christenson RH. Stroke Biomarkers: Progress and Challenges for Diagnosis, Prognosis, Differentiation, and Treatment. Clinical Chemistry 2010;56:21-33.

2. Lloyd-Jones D, Adams R, Carnethon M, De Simone G, Ferguson TB, Flegal K. Heart disease and stroke statistics 2009 update: a report from the American Heart Association Statistics Committee and Stroke Statistics Subcommittee. Circulation 2009;119:e21-181.

3. Adams HPJ, Bendixen BH, Kappelle LJ, Biller J, Love BB, Gordon DL, Marsh EE 3rd. Classification of subtype of acute ischemic stroke: definitions for use in a multicenter clinical trial TOAST. Trial of Org 10172 in Acute Stroke Treatment. Stroke 1993;24:3541.

4. Morgenstern LB, Lisabeth LD, Mecozzi AC, Smith MA, Longwell PJ, McFarling DA, Risser JM. A population-based study of acute stroke and TIA diagnosis. Neurology 2004;62: 895-900.

5. Hand PJ, Kwan J, Lindley RI, Dennis MS, Wardlaw JM. Distinguishing between stroke and mimic at the bedside: the brain attack study. Stroke 2006;37:769-75.
6. Adams HP Jr, Del Zoppo GJ, Alberts MJ, Bhatt DL, Brass L, Furlan A. Guidelines for the early management of adults with ischemic stroke: a guideline from the American Heart Association/ American Stroke Association Stroke Council, Clinical Cardiology Council, Cardiovascular Radiology and Intervention Council, and the Atherosclerotic Peripheral Vascular Disease and Quality of Care Outcomes in Research Interdisciplinary Working Groups: the American Academy of Neurology affirms the value of this guideline as an educational tool for neurologists. Circulation 2007;115:e478534.

7. Goldstein LB, Samsa GP. Reliability of the National Institutes of Health Stroke Scale: extension to non-neurologists in the context of a clinical trial. Stroke 1997;28:307-10.

8. Tan JC, Dillon WP, Liu S, Adler F, Smith WS, Wintermark M: Systematic comparison of perfusion-CT and CT-angiography in acute stroke patients. Ann Neurol 2007;61:533-43.

9. Kohrmann M, Schellinger PD. Acute stroke triage to intravenous thrombolysis and other therapies with advanced CT or MR imaging: pro MR imaging. Radiology 2009; 251:627-33.

10. Gonzalez RG, Schaefer PW, Buonanno FS, Schwamm LH, Budzik RF, Rordorf G. Diffusion-weighted MR imaging: diagnostic accuracy in patients imaged within 6 hours of stroke symptom onset. Radiology 1999;210:155-62.

11. Braunewell KH, Klein-Szanto AJ. Visinin-like proteins (VSNLs): interaction partners and emerging functions in signal transduction of a subfamily of neuronal Ca2 +-sensor proteins. Cell Tissue Res. 2009;335:301-16.

12. Reiber H, Felgenhauer K. Protein transfer at the blood cerebrospinal fluid barrier and the quantitation of the humoral immune response within the central nervous system. Clin Chim Acta. 1987;163:319-28.

13. Reynolds MA, Kirchick HJ, Dahlen JR, Anderberg JM, McPherson PH, Nakamura KK. Early biomarkers of stroke. Clin Chem 2003;49:1733-9.

14. Laskowitz DT, Blessing R, Floyd J, White WD, Lynch JR. Panel of biomarkers predicts stroke. Ann N Y Acad Sci 2005;1053:30.

15. Whiteley W, Tseng M-C, Sandercock P. Blood biomarkers in the diagnosis of ischemic stroke: a systematic review. Stroke 2008;39:2902-9.

16. Buttgereit J, Qadri F, Monti J, Langenickel TH, Dietz R, Braunewell KH, Bader M. Visinin-like protein 1 regulates natriuretic peptide receptor B in the heart. Regul Pept. 2010;161:51-7.

17. Gierke P, Zhao C, Bernstein HG, Noack C, Anand R, Heinemann $\mathrm{U}$, Braunewell $\mathrm{KH}$. Implication of neuronal $\mathrm{Ca} 2+$-sensor protein VILIP-1 in the glutamate hypothesis of schizophrenia. Neurobiol Dis. 2008;32:162-75. 